Discussion Paper No. 02-63

\title{
The Empirical Assessment of Technology Differences: Comparing the Comparable
}

Manuel Frondel and Christoph M. Schmidt

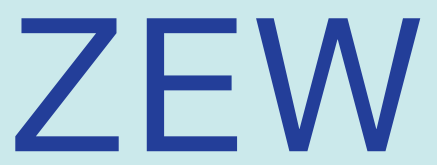

Zentrum für Europäische Wirtschaftsforschung GmbH

Centre for European Economic Research 
Discussion Paper No. 02-63

\title{
The Empirical Assessment of Technology Differences: Comparing the Comparable
}

\author{
Manuel Frondel and Christoph M. Schmidt
}

Download this ZEW Discussion Paper from our ftp server:

ftp://ftp.zew.de/pub/zew-docs/dp/dp0263.pdf

Die Discussion Papers dienen einer möglichst schnellen Verbreitung von neueren Forschungsarbeiten des ZEW. Die Beiträge liegen in alleiniger Verantwortung der Autoren und stellen nicht notwendigerweise die Meinung des ZEW dar.

Discussion Papers are intended to make results of ZEW research promptly available to other economists in order to encourage discussion and suggestions for revisions. The authors are solely responsible for the contents which do not necessarily represent the opinion of the ZEW. 
Non-technical Summary. One and the same production technology, which is arguably "close" to itself, displays similar substitution elasticities when economic circumstances are comparable. It seems natural, therefore, to classify two technologies as "close" when their substitution elasticities are similar under comparable economic circumstances. Following this reasoning, this paper compares technologies across space and time on the basis of factual and counterfactual substitution elasticities and argues that differences in estimated substitution elasticities should be decomposed into two counterfactual components. While the first component is designed to indicate how the ease of substitution is altered by varied economic circumstances, the second addresses the question of how technologies would compare under genuinely comparable situations. This decomposition is very much related to the prominent OAXACA-BLINDER decomposition in the analysis of wage differences. We illustrate our argument with the example of energy-price elasticities of capital before and after the oil crisis of the early 1970s. From our counterfactual analysis, it becomes transparent that substantial insight into the matter - questions of empirical inference notwithstanding - necessitates first of all the comparison of comparable situations, designed to reflect genuine differences in technologies. With respect to the comparison of energy-price elasticities of capital across various studies, specifically, this counterfactual perspective implies that the question of substitutability of capital and energy cannot be answered without explicit regard to economic circumstances, specifically to factor prices. 


\title{
The Empirical Assessment of Technology Differences: Comparing the Comparable.
}

Manuel Frondel, Centre for European Economic Research, Mannheim,

Christoph M. Schmidt, RWI, Essen, IZA, Bonn, and CEPR, London

October 2002

\begin{abstract}
This paper compares technologies across space and time on the basis of factual and counterfactual substitution elasticities and argues that differences in estimated substitution elasticities should be decomposed into two counterfactual components. While the first component is designed to indicate how the ease of substitution is altered by varied economic circumstances, the second addresses the question of how technologies would compare under genuinely comparable situations. This argument is illustrated by the example of energy-price elasticities of capital before and after the oil crisis of the early 1970s.
\end{abstract}

JEL classification: C3, D2.

Key words: Counterfactuals, Substitutability, Translog Cost Function.

Correspondence: Prof. Dr. Christoph M. Schmidt (Ph. D.), Rheinisch-Westfälisches Institut für Wirtschaftsforschung, Hohenzollernstr. 1-3, D-45128 Essen. E-mail: Schmidt@rwi-essen.de. We would like to thank Dr. Andreas Ziegler for his careful reading of the manuscript. Financial support by the Centre for European Economic Research is gratefully acknowledged. 


\section{Introduction}

In principle, the question of whether production units operate under relatively similar or vastly different technologies lies at the core of economic reasoning. Theoretically, it is easy to agree that the closeness of substitution elasticities is at issue. Yet, its empirical assessment is far from straightforward: Modern approaches to the empirical analysis of the production of goods and services typically acknowledge the possibility that the ease of substituting one factor for another might differ according to the circumstances under which this question is addressed. Specifically, for production technologies represented by flexible functional forms such as translog production or cost functions, substitution elasticities vary systematically with factor prices, indicating that it is more and more difficult to substitute for a factor whose price becomes smaller and smaller. Since this property is intuitively appealing, for most applied researchers the choice of such a flexible functional form seems to be particularly advisable.

When comparing production technologies across space and time, the very fact that substitution elasticities tend to vary with economic conditions such as prices necessitates the clarification of whether observed differences reflect genuine discrepancies in technology or simply different economic circumstances. Surprisingly little thought has been given to this question, though. This is all the more remarkable since it is well-accepted that one and the same technology displays different substitution elasticities when factor prices are altered, although it is arguably "close" to itself. Thus, it seems natural to classify two technologies as "close" when their substitution elasticities are similar under comparable economic circumstances, specifically for comparable factor prices - even if these circumstances are not observed in the data.

This paper compares technologies across space and time on the basis of actually estimated (factual) and counterfactual substitution elasticities and argues that differences in estimated substitution elasticities should be decomposed into two counterfactual components. While the first component is designed to indicate how the ease of substitution is altered by the observed variation in economic circumstances, the second component addresses the question of how the technologies in question would compare under genuinely 
comparable situations. This decomposition is very much related to the prominent OAXACABLINDER decomposition in the analysis of wage differences - seminal papers are BLINDER (1973), Oaxaca (1973), and OaxacA-Ransom (1994). Since this paper's major argument is on matters of identification, we will proceed as if translog coefficients were estimated with infinite precision.

We document the relevance of our argument with the illustrative example of energyprice elasticities of capital before and after the oil crisis of the early 1970s. From our counterfactual analysis, it becomes transparent that - notwithstanding questions of empirical inference - substantial insight into the empirical assessment of technology differences first necessitates the comparison of comparable situations. With respect to the famous capitalenergy controversy, for instance, this counterfactual perspective implies that the question of substitutability of capital and energy cannot be answered without explicit regard to economic circumstances, specifically to factor prices: A part of the discrepancies in the empirical results of this controversy - see FRONDEL and SCHMIDT (2002) for a straightforward explanation for static translog approaches - might have been due to the comparison of incomparable economic situations, rather than reflecting genuine differences in technologies.

Section 2 deals with the construction of counterfactual situations for static translog approaches. In Section 3, we employ U. S. manufacturing (1958-1996) data covering the years of the oil crisis of the 1970s, and calculate counterfactual energy-price elasticities in order to compare them to actually estimated elasticities. The last section concludes.

\section{Static Translog Cost Functions}

A typical static translog study departs from the assumption that there exists in manufacturing a homothetic, twice differentiable aggregate translog cost function of the form (see e. g. TAKAYAMA 1985:148)

$$
\ln C\left(p_{1}, \ldots, p_{I}, Y\right)=\beta_{0}+\beta_{Y} \ln Y+\sum_{i=1}^{I} \beta_{i} \ln p_{i}+\frac{1}{2} \sum_{i, j=1}^{I, I} \beta_{i j} \ln p_{i} \ln p_{j},
$$

where $p_{i}$ denotes the price of input $i$ and $Y$ aggregate output. Symmetry of $\beta_{i j}$ is typically imposed a priori. Applying SHEPHARD's Lemma, $x_{i}=\frac{\partial C}{\partial p_{i}}$, and differentiating (1) logarith- 
mically, one can derive an equation system of linear expressions for the share $s_{i}$ of overall cost attributable to each factor $i(i=1, \ldots, I)$ :

$$
s_{i}:=\frac{x_{i} p_{i}}{C}=\frac{\partial \ln C}{\partial \ln p_{i}}=\beta_{i}+\sum_{j=1}^{I} \beta_{i j} \ln p_{j} .
$$

Unknown parameters, summarized by vector $\beta:=\left(\beta_{1}, \ldots, \beta_{i}, \ldots \beta_{I}, \beta_{11}, \ldots, \beta_{i j}, \ldots, \beta_{I I}\right)^{\prime}$, can be estimated from a stochastic version of this cost-share system, where prices are assumed to be exogenous and each equation additionally contains a vector $\varepsilon$ of additive orthogonal stochastic disturbances. For estimation purposes, prices $p^{t}:=\left(p_{1}^{t}, \ldots, p_{I}^{t}\right)^{\prime}$ are typically normalized to unity for the first year of the data range: $p^{1}=(1, \ldots, 1)^{\prime}$. This specific choice is inconsequential, though, for the estimation of substitution elasticities. Take the specific example of cross-price elasticities estimated from translog approaches, which are our focus in the empirical application. First, the alternative normalization $\tilde{p}^{t_{1}}:=\left(\tilde{p}_{1}^{t_{1}}, \ldots, \tilde{p}_{I}^{t_{1}}\right)^{\prime}=(1, \ldots, 1)^{\prime}$ of prices for any arbitrary year $t_{1}$ leaves the estimates of all second-order coefficients $\beta_{i j}$ unchanged: For $t_{2}$, for instance, equation system (2) reads

$$
s_{i}^{t_{2}}=\beta_{i}+\sum_{j=1}^{I} \beta_{i j} \ln p_{j}^{t_{2}}
$$

while, with $\tilde{p}^{t_{2}}=\left(\tilde{p}_{1}^{t_{2}}, \ldots, \tilde{p}_{I}^{t_{2}}\right)^{\prime}:=\left(p_{1}^{t_{2}} / p_{1}^{t_{1}}, \ldots, p_{I}^{t_{2}} / p_{I}^{t_{1}}\right)^{\prime}$, it is

$$
s_{i}^{t_{2}}=\tilde{\beta}_{i}+\sum_{j=1}^{I} \tilde{\beta}_{i j} \ln \tilde{p}_{j}^{t_{2}}=\tilde{\beta}_{i}+\sum_{j=1}^{I} \tilde{\beta}_{i j} \ln \left(p_{j}^{t_{2}} / p_{j}^{t_{1}}\right)=\tilde{\beta}_{i}-\sum_{j=1}^{I} \tilde{\beta}_{i j} \ln p_{j}^{t_{1}}+\sum_{j=1}^{I} \tilde{\beta}_{i j} \ln p_{j}^{t_{2}} .
$$

Since the expressions (3) and (4) hold for any price vector $p^{t_{2}}$ in the relevant range, specifically for $p^{t_{2}}=(1, \ldots, 1)^{\prime}, \tilde{\beta}_{i j}$ has to equal $\beta_{i j}$ for all $i$ and $j$, whereas

$$
\beta_{i}=\tilde{\beta}_{i}-\sum_{j=1}^{I} \tilde{\beta}_{i j} \ln p_{j}^{t_{1}}
$$

and hence $\beta_{i} \neq \tilde{\beta}_{i}$, in general. That is, the expressions (3) and (4) only differ in the constant term and only the estimates of first-order coefficients depend upon the specific normalization of prices. Second, it becomes obvious from (3) and (4), and the interrelations between both parameter sets $\beta$ and $\tilde{\beta}$, that estimates of cost shares $s_{i}$ are the same regardless of the concrete normalization.

Third, in the further analysis, the exposition focuses on cross-price elasticities $\eta_{x_{i} p_{j}}$, specifically on $\eta_{K p_{E}}$, the energy-price elasticity of capital. For translog cost functions (1), the 
analytical expression of any cross-price elasticity $\eta_{x_{i} p_{j}}$ can be derived by differentiating share equation (2) $\operatorname{logarithmically~with~respect~to~} p_{j}$, and using $\frac{\partial \ln C}{\partial \ln p_{j}}=s_{j}$ as well as $\frac{\partial \ln p_{i}}{\partial \ln p_{j}}=0$ :

$$
\eta_{x_{i} p_{j}}:=\frac{\partial \ln x_{i}}{\partial \ln p_{j}}=\frac{\partial \ln }{\partial \ln p_{j}}\left(\frac{s_{i} C}{p_{i}}\right)=\frac{\partial \ln s_{i}}{\partial \ln p_{j}}+\frac{\partial \ln C}{\partial \ln p_{j}}-\frac{\partial \ln p_{i}}{\partial \ln p_{j}}=\frac{\beta_{i j}}{s_{i}}+s_{j} .
$$

Hence, because estimates of both, cost shares and second-order coefficients, are the same irrespective of the concrete price-normalization, so are estimates of the cross-price elasticities given by (6).

In other words, merely relative prices matter in the estimation of these elasticities - anything else would defy economic intuition. Thus, two researchers analyzing the same data set but using different normalizations would obtain different coefficient estimates but identical estimates of cross-price elasticities. Yet, while questions of price normalization are irrelevant for the analysis of a single empirical situation, they are at issue when several studies are compared on the basis of counterfactual elasticities - a task that is addressed in Section 3.

Obviously, the cost shares $s_{i}$ and $s_{j}$ of both factors $i$ and $j$ are pivotal elements of the cross-price elasticity $\eta_{x_{i} p_{j}}$. If translog cost function (1) specializes to the CoBB-DougLAS function, that is, if $\beta_{i j}=0$ for all $i, j$, the cross-price elasticity $\eta_{x_{i} p_{j}}$ equals $s_{j}$, the constant cost share of factor $j$. Expression (6) demonstrates that the cost share $s_{j}$ of factor $j$ sets the empirical benchmark for the cross-price elasticity $\eta_{x_{i} p_{j}}$, and moreover that the estimated cross-price elasticity $\eta_{x_{i} p_{j}}$ will be closer to $s_{j}$ the larger the cost share $s_{i}$ of factor $i$ relative to the second-order coefficient $\beta_{i j}$ is. For the factors energy and capital, in particular, it is the cost share $s_{E}$ of energy which represents the benchmark for estimates of energy-price elasticities $\eta_{K p_{E}}$ of capital. FRONDEL and SCHMIDT (2002) provide ample evidence for the empirical relevance of this straightforward cost-share argument in the context of the capitalenergy controversy. In sum, estimated cross-price elasticities tend to reflect the particular cost shares and hence the economic circumstances under which these estimates are derived.

In situations in which the task is to assess differences in distinct production technologies across space and time, this observation has drastic consequences. One and the same production technology that is arguably "close" to itself displays different substitution elasticities when economic circumstances, that is prices and hence cost shares, are altered. What part of 
any observed difference across substitution elasticities should then be attributed to different economic circumstances, and what to genuine discrepancies in technologies? One possible route towards a sensible comparison of technologies is the examination of the respective coefficients estimated for a common flexible functional form.

As our discussion of normalization issues has demonstrated, such coefficients usually do not have any direct economic interpretation - as opposed to parameters like substitution elasticities derived from these coefficients. Moreover, this route provides a serious practical difficulty. Which discrepancy across the typically quite large number of coefficients is to be taken more seriously? It is the very purpose of empirical analyses to condense the multitude of coefficients into summary parameters such as substitution elasticities. It seems natural, therefore, to classify two technologies as "close" when their substitution elasticities are similar under comparable economic circumstances. Consequently, we suggest the comparison of distinct production technologies on the basis of substitution elasticities in comparable situations, that is, when relative prices are held constant. In effect, we suggest constructing counterfactual substitution elasticities.

\section{The Construction of Counterfactual Situations}

In the further discussion, we retain our focus on translog approaches, and on the energy-price elasticities of capital. In translog studies, the analytic expression for these elasticities,

$$
\eta_{K p_{E}}(\beta, p):=\frac{\beta_{K E}}{s_{K}}+s_{E}=\frac{\beta_{K E}}{\left\{\beta_{K}+\sum_{j=1}^{I} \beta_{K j} \ln p_{j}\right\}}+\left\{\beta_{E}+\sum_{j=1}^{I} \beta_{E j} \ln p_{j}\right\},
$$

reveals that they are implicitly assumed to be functions of only two components: The underlying production technology, on the one hand, being condensed in the first- and second-order coefficients $\beta:=\left(\beta_{1}, \ldots, \beta_{K}, \beta_{E}, \ldots, \beta_{J}, \beta_{11}, \ldots, \beta_{K E}, \ldots, \beta_{I I}\right)^{\prime}$ to be estimated, and the observable prices $p:=\left(p_{1}, \ldots, p_{K}, p_{E}, \ldots, p_{J}\right)^{\prime}$ for capital, energy and other production factors, on the other. The maintained hypothesis is that parameters $\beta$ remain the same when economic conditions change; this constancy of the translog parameters $\beta$ is precisely the assumption underlying their estimation. While the coefficients $\beta$ are constant parameters to be uncovered in the empirical estimation, the parameters of interest - the substitution elasticities - differ according to the circumstances, that is, the factor prices under which they are determined. 
For a given technology, these properties make it quite straightforward to infer on the ease of substitution of the factors energy and capital in a multitude of situations - those observed and hypothetical situations. All that is altered across situations are the associated relative factor prices. (Note that the price level is completely irrelevant for our question of interest.) In effect, using counterfactual relative prices $p^{1}$ instead of observed relative prices allows us to investigate which energy-price elasticities of capital would result if the prices, specifically those of capital and energy, were different from actual prices, while the technology in use remains the same.

This reasoning allows us to quantitatively assess differences in distinct production technologies - as captured by substitution possibilities. Specifically, it might well be that $\eta_{K p_{E}}\left(\beta^{0}, p^{0}\right)$, the substitution elasticity describing one study comprehensively, and $\eta_{K p_{E}}\left(\beta^{1}, p^{1}\right)$, the corresponding elasticity capturing the situation observed in another study, are quite different.

Yet, these two studies might merely uncover how one and the same translog technology produces quite different results in the situations in which relative price vectors differ. Nevertheless, in the counterfactual situation in which the prices observed in the first study, say, were to arise in the second study, the resulting substitution elasticities might be similar,

$$
\eta_{K p_{E}}\left(\beta^{1}, p^{0}\right) \approx \eta_{K p_{E}}\left(\beta^{0}, p^{0}\right)
$$

In the special case of a CoBB-Douglas technology $\left(\beta_{i j}=0\right.$ for all $i$ and $\left.j\right)$, where according to expression (7) $\eta_{K p_{E}}=s_{E}$ and $s_{E}=\frac{\ln C}{\ln p_{E}}=\beta_{E}$ (see equation (2)), energy-price elasticities of capital are similar only when the output elasticities of energy and, hence, cost shares of energy are similar. Thus, the particular price vector arising in either study can only play a role for the appropriate construction of comparable substitution elasticities when the technologies deviate from the CoBB-Douglas case.

This insight suggests the translation of the famous OAXACA-BLINDER decomposition of wage differences to the case of translog cost functions,

$$
\eta_{K p_{E}}\left(\beta^{1}, p^{1}\right)-\eta_{K p_{E}}\left(\beta^{0}, p^{0}\right)=\left[\eta_{K p_{E}}\left(\beta^{1}, p^{1}\right)-\eta_{K p_{E}}\left(\beta^{1}, p^{0}\right)\right]+\left[\eta_{K p_{E}}\left(\beta^{1}, p^{0}\right)-\eta_{K p_{E}}\left(\beta^{0}, p^{0}\right)\right] .
$$

The first term in parentheses on the right-hand side of decomposition (9) captures the variation in elasticities as circumstances on the economic environment change for the same 
technology, while the second term in parentheses, holding prices fixed, captures genuine differences in structure or technology.

It is well known for OAXACA-BLINDER decompositions that the decomposition into these two additive terms is not unique. It varies with the particular choice of baseline technology $-\beta^{1}$ in the first term of decomposition (9) - and the corresponding choice of baseline circumstances $-p^{0}$ in the second term of decomposition (9). Equally plausible might have been the alternative decomposition:

$\eta_{K p_{E}}\left(\beta^{1}, p^{1}\right)-\eta_{K p_{E}}\left(\beta^{0}, p^{0}\right)=\left[\eta_{K p_{E}}\left(\beta^{1}, p^{1}\right)-\eta_{K p_{E}}\left(\beta^{0}, p^{1}\right)\right]+\left[\eta_{K p_{E}}\left(\beta^{0}, p^{1}\right)-\eta_{K p_{E}}\left(\beta^{0}, p^{0}\right)\right]$.

More generally, it is the respective counterfactual question of economic interest - here, the adequate choice of counterfactual prices $p^{*}$ specifically - that suggests the appropriate decomposition:

$$
\begin{aligned}
\eta_{K p_{E}}\left(\beta^{1}, p^{1}\right)-\eta_{K p_{E}}\left(\beta^{0}, p^{0}\right)= & {\left[\eta_{K p_{E}}\left(\beta^{1}, p^{1}\right)-\eta_{K p_{E}}\left(\beta^{1}, p^{*}\right)\right]+\left[\eta_{K p_{E}}\left(\beta^{1}, p^{*}\right)-\eta_{K p_{E}}\left(\beta^{0}, p^{*}\right)\right] } \\
& +\left[\eta_{K p_{E}}\left(\beta^{0}, p^{*}\right)-\eta_{K p_{E}}\left(\beta^{0}, p^{0}\right)\right]
\end{aligned}
$$

Ultimately, an assessment of the similarity between technologies requires the comparison of substitution elasticities for a baseline or benchmark situation on the basis of standardized prices $p^{*}$. Decomposition (11) suggests the calculation of the difference $\eta_{K p_{E}}\left(\beta_{K E}^{1}, p^{*}\right)-$ $\eta_{K p_{E}}\left(\beta^{0}, p^{*}\right)$ to provide this comparison.

\section{Counterfactual Capital-Energy Elasticities}

To provide an illustration, we present an example characterized by significant technology changes. As e. g. Jorgenson and Stiroh (2000), we employ Jorgenson's time-series data set $^{1}$ of U. S. manufacturing (1958-1996), which encompasses the years of the oil crisis of the early 1970s. Of the available 35 sectors, we concentrate on time-series data for the primary metals sector, one of the most energy-intensive sectors, and split up this data set into two subsamples. The first subsample (1958-1973) covers the baseline period " 0 " before

\footnotetext{
${ }^{1}$ This data set is accessible via internet - see Prof. Jorgenson's homepage: http://post.economics.harvard.edu/faculty/jorgenson/
} 
the first serious oil crisis of 1973/1974, while the second (1974-1996) includes the phase "1" thereafter. Thus, one might expect substantial differences to emerge between the technologies prevailing in both periods.

To address this issue, we provide two sets of coefficient estimates, each based on one of the subsamples, respectively. We estimate the corresponding share equations via ML, with symmetry and homogeneity imposed, while prices are normalized to the year 1992. On the basis of the coefficient estimates, we then construct estimates of the implied - "factual" and counterfactual - energy-price elasticities of capital displayed in Table $\mathbf{1}$.

Table 1: "Factual" and Counterfactual Energy-Price Elasticities with respect to Capital for the U. S. Primary Metals Sector.

\begin{tabular}{|c|c|c|c|c|c|c|c|}
\hline & \multicolumn{3}{|c|}{ Period "0": 1958-1973 } & & \multicolumn{3}{|c|}{ Period "1": 1974-1996 } \\
\hline & 1967 & 1970 & 1973 & & 1974 & 1977 & 1980 \\
\hline \multicolumn{8}{|c|}{ Price Indices $\left(p^{1992}=(1,1,1,1)^{\prime}\right)$ : } \\
\hline$p_{K}$ & 0.377 & 0.281 & 0.393 & & 0.614 & 0.374 & 0.521 \\
\hline$p_{L}$ & 0.150 & 0.181 & 0.234 & & 0.271 & 0.423 & 0.521 \\
\hline$p_{E}$ & 0.171 & 0.193 & 0.244 & & 0.340 & 0.504 & 0.758 \\
\hline$p_{M}$ & 0.280 & 0.325 & 0.367 & & 0.451 & 0.587 & 0.808 \\
\hline \multicolumn{8}{|c|}{ "Factual" Cross-Price Elasticities: } \\
\hline$\eta_{K p_{E}}\left(\beta^{0}, p^{0}\right)$ & -0.007 & -0.022 & -0.013 & $\eta_{K p_{E}}\left(\beta^{1}, p^{1}\right)$ & 0.104 & 0.147 & 0.164 \\
\hline \multicolumn{8}{|c|}{ Counterfactual Cross-Price Elasticities: } \\
\hline \multicolumn{3}{|c|}{$\eta_{K p_{E}}\left(\beta^{0}, p^{1}=p^{1980}\right)$} & -0.034 & \multicolumn{3}{|c|}{$\eta_{K p_{E}}\left(\beta^{1}, p^{0}=p^{1967}\right)$} & 0.071 \\
\hline \multicolumn{3}{|c|}{$\eta_{K p_{E}}\left(\beta^{0}, p^{*}=(1,1,1,1)^{\prime}\right)$} & -0.012 & \multicolumn{3}{|c|}{$\eta_{K p_{E}}\left(\beta^{1}, p^{*}=(1,1,1,1)^{\prime}\right)$} & 0.143 \\
\hline
\end{tabular}

The qualitative character of the factual substitution elasticities has changed substantially over time. In the period 1958-1973, the corresponding estimates were slightly negative - capital and energy were to be classified as complements. ${ }^{2}$ By contrast, the substitution elasticities for the post-oil crises period clearly indicate a substitution relationship between capital and energy. Yet, the relative prices of energy and other production factors have developed quite differently over time. In particular, the prices of both energy and materials accelerated disproportionately after the oil crisis. To what extent, we therefore have to ask,

\footnotetext{
${ }^{2}$ Since these figures are presented for illustrative purposes regarding our fundamental argument for comparing genuinely comparable situations, we deliberately abstain from sampling issues here. Whether or not estimates are estimated precisely is completely irrelevant here, albeit not for the literature on capital and energy substitution, where it has been ignored - see Frondel and Schmidt (2002).
} 
are the discrepancies in elasticity estimates between pre- and post-oil-crisis periods due to altered economic circumstances?

To answer this question, we provide a comparison of comparable situations across both periods along the lines of decomposition (11). First, we construct counterfactual elasticities for each of the sub-periods, with relative prices of 1980 applied to the coefficients the earlier period, and of 1967 to those of the latter period. While being less accentuated, these estimates document that the fundamental shift in the nature of production is genuine, not merely a reflection of altered relative prices. This result is confirmed when applying the common price vector $p^{*}=p^{1992}=(1,1,1,1)^{\prime}$. Had we attempted to judge the issue on the basis of factual substitution elasticities, no such statement would have been possible.

Finally, we have demonstrated in Section 2 that the specific price normalization is irrelevant for the estimation results within each subsample. Yet, questions of price normalization are at issue when estimation results of distinct studies are compared on the basis of counterfactual elasticities. It is straightforward to harmonize price indices between the two studies by imposing a common baseline period, as 1992 was in our illustration. The coefficient estimates, however, alos need to be transformed to reflect this new baseline vector. Specifically, one has to apply the normalizing price vector to the invariant second-order coefficient estimates, and to alter first-order coefficient estimates according to expression (5). After this preparatory stage, the construction of counterfactual elasticities can proceed as shown.

\section{Summary and Conclusion}

Comparisons of technologies across space and time deliberately focus on the ease of substitution among production factors. Yet, one and the same technology might produce quite different substitution elasticities merely as a consequence of discrepancies in economic situations. The question, therefore, is how to compare substitution elasticities provided by distinct empirical studies. By using a translog specification and the example of energy-price elasticity estimates of capital for U. S. manufacturing before and after the oil crises of the early 1970s, this paper exemplifies a possible way for comparing production technologies in 
empirical work. It is argued that differences in estimated substitution elasticities should be decomposed into two counterfactual components. While the first component is designed to indicate how the ease of substitution is altered by varied economic circumstances, the second addresses the question of how technologies would compare under genuinely comparable situations.

Our results indicate that the construction and comparison of counterfactual elasticities to "factual" elasticity estimates on the basis of decompositions (9) - (11) should be at the heart of any sensible empirical assessment of technology differences. This importance of creating comparable situations by constructing counterfactuals is a perspective commonly adopted in the modern literature on econometric evaluation. Our illustrative example points out that a small part of the controversy in the literature about complementarity or substitutability of capital and energy, specifically, might have been a consequence of comparing incomparable situations. Quite generally, apparent differences in substitution elasticities might originate partly from the confrontation with different price indices, not from genuine differences in technology. 


\section{References}

Blinder A. S. (1997): Wage Discrimination: Reduced Form and Structural Estimates, Journal of Human Resources, Vol. 8:436-455.

Frondel M. and C. M. Schmidt (2002): The Capital-Energy Controversy: An Artifact of Cost Shares? The Energy Journal, Vol. 23, No. 3:53-79.

Jorgenson, D. W. and K. J. Stiroh (2000): Raising the Speed Limit: U.S. Economic Growth in the Information Age. Brookings Papers on Economic Activity, Vol. 1:125-211.

OAXacA R. L. (1973): Male-Female Wage Differentials in Urban Labor Markets. International Economic Review, Vol. 9:693-709.

Oaxaca R. L. and M. R. Ransom (1994): On Discrimination and the Decomposition of Wage Differentials. In: Neuman S. and Silber J. : The Econometrics of Labor Market Segregation and Discrimination, Journal of Econometrics, Vol. 61:5-21.

Takayama A. (1985): Mathematical Economics. Second edition. Cambridge University Press, Cambridge, MA. 